VOL. $61(2000) \quad[173-174]$

\title{
Nonmetrisable manifolds
}

\author{
Sina GreEnwood
}

Nyikos [1] has defined a tree, denoted $\Upsilon$, associated with any given Type I space. This thesis examines the properties of an $\Upsilon$-tree if the space is a Type I nonmetrisable manifold. It is shown that a tree, $T$, is an $\Upsilon$-tree of a Type I manifold if and only if $T$ is a well-pruned $\omega_{1}$-tree. Furthermore, if $T$ is any well-pruned $\omega_{1}$-tree, there are $2^{\aleph_{1}}$ different Type I manifolds for which $T$ is the $\Upsilon$-tree. The relationships between the properties of a Type I manifold and the properties of its $\Upsilon$-tree are examined. It is shown that whenever a Type I manifold contains a copy of $\omega_{1}$, its $\Upsilon$-tree must contain an uncountable branch. The thesis then addresses the problem of whether or not an arbitrary tree $T$ admits a Type I manifold which is $\omega_{1}$-compact. If $T$ does not contain an uncountable antichain, or a Suslin subtree, then there exists a Type I manifold with $\Upsilon$-tree $T$. If $T$ contains an uncountable antichain, then whether there exists an $\omega_{1}$-compact Type I manifold with $\Upsilon$-tree $T$ is undecidable. (*) implies there does not exist such a tree while $\diamond$ implies that there does. If we assume $\boldsymbol{\beta}^{+}$, then at least one such manifold exists. $\diamond$ also implies that if $T$ contains a Suslin subtree, then there exists an $\omega_{1}$-compact manifold with $\Upsilon$-tree $T$.

Nyikos has recently defined a Type II space. We may associate an $\Upsilon \Upsilon \Upsilon$-tree with such a space. This thesis shows that a Tree, $T$, admits a Type II manifold if and only if $T$ has height not greater than $\omega_{1}$, and each level has cardinality no greater than $\mathrm{c}$.

The final chapter examines the relationship between microbundles and fibre bundles over nonmetrisable manifolds. In 1964 Milnor [2] defined the notion of a microbunble. He ceased developing the theory of microbundles when later in the same year Kister showed that a microbundle over a metrisable manifold is equivalent to a fibre bundle. This thesis proves that the tangent microbundle over a manifold is a fibre bundle if and only if the manifold is metrisable. As a consequence of this we obtain further properties equivalent to metrisability in a manifold.

Received 6th September, 1999

Thesis submitted to the University of Auckland, March 1999. Degree approved, July 1999. Supervisors: Professor David Gauld and Dr David McIntyre.

Copyright Clearance Centre, Inc. Serial-fee code: 0004-9727/00 \$A2.00+0.00. 


\section{REFERENCES}

[1] P. Nyikos, 'The theory of nonmetrizable manifolds', in Handbook of Set-theoretic Topology, (K. Kunen and J.E. Vaughan, Editors) (North-Holland, Amsterdam, New York, 1984), pp. 633-684.

[2] J. Milnor, 'Microbundles Part I', Topology 3 (1964), 53-80.

Department of Mathematics

The University of Auckland

Private Bag 92019

Auckland

New Zealand 